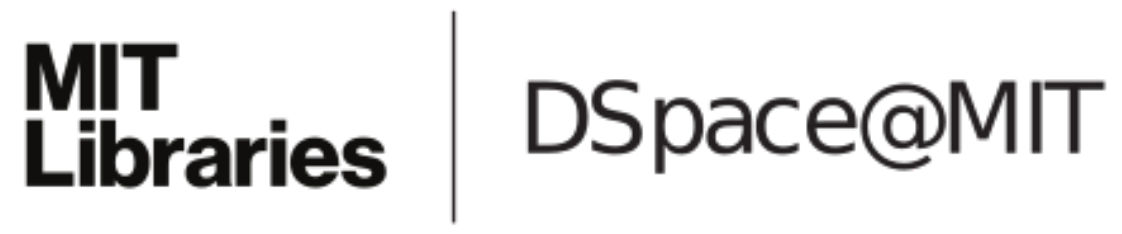

\author{
MIT Open Access Articles
}

Basal ganglia output to the thalamus: still a paradox

The MIT Faculty has made this article openly available. Please share how this access benefits you. Your story matters.

Citation: Goldberg, Jesse H., Michael A. Farries, and Michale S. Fee. "Basal Ganglia Output to the Thalamus: Still a Paradox." Trends in Neurosciences 36, no. 12 (December 2013): 695-705.

As Published: http://dx.doi.org/10.1016/j.tins.2013.09.001

Publisher: Elsevier

Persistent URL: http://hdl.handle.net/1721.1/102409

Version: Author's final manuscript: final author's manuscript post peer review, without publisher's formatting or copy editing

Terms of use: Creative Commons Attribution-NonCommercial-NoDerivs License 


\title{
Basal ganglia output to the thalamus: still a paradox
}

\author{
Jesse H. Goldberg ${ }^{1}$, Michael A. Farries ${ }^{2}$, and Michale S. Fee ${ }^{1}$ \\ ${ }^{1}$ McGovern Institute for Brain Research, Department of Brain and Cognitive Sciences, \\ Massachusetts Institute of Technology, Cambridge, MA \\ ${ }^{2}$ Department of Biology, University of Texas at San Antonio, San Antonio, TX
}

\begin{abstract}
The basal ganglia $(\mathrm{BG})$ recipient thalamus controls motor output but it remains unclear how its activity is regulated. Several studies report that thalamic activation occurs via disinhibition during pauses in the firing of inhibitory pallidal inputs from the BG. Other studies indicate that thalamic spiking is triggered by pallidal inputs via post-inhibitory 'rebound' calcium spikes. Finally excitatory cortical inputs can drive thalamic activity, which becomes entrained, or time-locked, to pallidal spikes. We present a unifying framework where these seemingly distinct results arise from a continuum of thalamic firing 'modes' controlled by excitatory inputs. We provide a mechanistic explanation for paradoxical pallidothalamic coactivations observed during behavior and raise new questions of what information is integrated in the thalamus to control behavior.
\end{abstract}

\section{Keywords}

Thalamus; cortex; basal ganglia; behavior

\section{Introduction}

The basal ganglia (BG) integrates information from a wide array of cortical, thalamic and brainstem inputs, and subsequently influences motor circuits through two major output pathways-a direct projection to brainstem circuitry, and a distinct projection to several nuclei in the motor thalamus [1-3]. Motor thalamus in turn projects widely to frontal and motor cortical areas (Figure 1A) [4, 5]. While much progress has been made in understanding how the BG may influence actions through the projection to behaviorgenerating circuits in the brainstem [6-8], less is known about how the basal ganglia influences motor behavior and learning through the thalamocortical output pathway $[9,10]$.

The BG-thalamic circuit is evolutionarily conserved [11, 12] (Figure 1). Across a wide range of behavioral paradigms and mammalian model systems thalamic lesions cause severe deficits in motor initiation, control and learning [13-17]. Furthermore, most thalamic neurons exhibit behavior-locked firing patterns, including brisk peaks in activity immediately prior to movement onsets (Figure 2B) [18-22]. We have recently found similar results in songbirds. Lesions of a song-related thalamic nucleus (DLM, dorsolateral nucleus

(c) 2013 Elsevier Ltd. All rights reserved.

Correspondence to: Jesse H. Goldberg.

*Current address: Department of Neurobiology and Behavior, Cornell University, Ithaca, NY

Publisher's Disclaimer: This is a PDF file of an unedited manuscript that has been accepted for publication. As a service to our customers we are providing this early version of the manuscript. The manuscript will undergo copyediting, typesetting, and review of the resulting proof before it is published in its final citable form. Please note that during the production process errors may be discovered which could affect the content, and all legal disclaimers that apply to the journal pertain. 
of the anterior thalamus) abolish exploratory vocalizations (babbling) and learning in juvenile birds [23]. Furthermore, DLM neurons exhibit pronounced peaks in firing immediately prior to syllable onsets during vocal babbling (Figure 2B) [24]. Together, these findings support the idea that the BG-recipient thalamus plays a critical role in activating motor cortical areas that generate movement or - in the songbird - that generate exploratory vocal gestures.

Here we aim to clarify how thalamus integrates inhibitory inputs from the BG and excitatory inputs from cortex. First, we briefly review what may have been considered three mutually exclusive perspectives of BG-thalamic transmission: disinhibition, rebound and entrainment. Next, we highlight recent mathematical simulations showing that rebound, disinhibition and entrainment are not actually incompatible aspects of thalamic function. Instead, they arise from distinct firing states, or 'modes' of operation of the thalamic neuron. We use the term 'mode' to indicate a case where a single neuron can exhibit distinct firing patterns depending on conditions [25], and we suggest that neurons in the BG-recipient thalamus operate in rebound, gating, or entrainment mode depending on their excitability.

Excitability, in turn could be controlled by the level of activation provided by neuromodulatory or cortical inputs. Finally, we synthesize recent findings from songbird, rodent and primate model systems-all of which point to the BG as a site where context, reward, and motor signals are integrated to produce learning. Notably, we highlight that, to our knowledge, none of these model systems has identified a role for the corticothalamic projection in these functions, and we propose testable hypotheses for future studies.

\section{BG-Thalamic Disinhibition and Gating}

What are the origins of thalamic premotor signals? One simple yet enduring idea is based on the observation that BG output neurons in the internal segment of the pallidum (GPi) and the substantia nigra pars reticulata (SNr) are GABAergic and tonically active, and they exert powerful inhibition of thalamic activity [25-27]. This inhibition is thought to suppress thalamic firing, thereby preventing movement initiation [28-30]. (For simplicity, we will lump GPi and SNr outputs together, and refer to both as 'pallidal', but there may be important differences in the way GPi and $\mathrm{SNr}$ outputs act on their thalamic targets).

Of course, the pallidal inhibition of thalamic activity is not fixed, but can be modulated by one or more inputs onto pallidal neurons. For example, activation of glutamatergic neurons in the subthalamic nucleus or of indirect pathway striatal neurons can cause an increase in tonic spiking of pallidal neurons, thus increasing the inhibition of the thalamus [31, 32] (Figure 1). Alternatively, activation of direct-pathway striatal neurons can cause a 'pause' in the tonic spiking of pallidal neurons, releasing the thalamic neurons from inhibition [33-36]. This process, referred to as 'disinhibition', produces a thalamic firing pattern that is typically depicted as the inverse of the pallidal firing rate: low thalamic activity results from high tonic pallidal firing, and high thalamic activity results from 'pauses' in pallidal input (Figure 1; Figure 2A).

There are also direct excitatory inputs to the BG-recipient thalamus, which arise from the cerebral cortex [4, 38-42]. Thus, motor thalamus need not simply 'invert' pallidal firing rate. Rather, the excitatory cortical drive may represent a signal to be transmitted to frontal and motor cortical targets [43]. In this view, high rates of tonic pallidal firing could block this signal, while pallidal pauses would open the thalamic 'gate,' allowing corticothalamocortical signals to pass [43]. This gating mechanism endows BG outputs with temporal control of thalamic transmission without necessarily defining the details of the transmitted signal [33, 44].

Trends Neurosci. Author manuscript; available in PMC 2014 December 01. 
A simple understanding of disease pathogenesis and motor control emerges from pallidothalamic disinhibition and gating [46]. First, hypokinetic diseases such as Parkinson's are thought to result from excess pallidal suppression of thalamus, and the subsequent inability to initiate movement. Hyperkinetic diseases such as Huntington's may arise from insufficient pallido-thalamic suppression, resulting in thalamic hyperactivity and movement excess [31]. Second, direct and indirect BG pathways have opposing actions on movement: activation of direct pathway medium spiny neurons (MSNs) should promote movement and relieve Parkinsonism, while activation of indirect pathway MSNs-which indirectly activate pallidal outputs-should suppress movement initiation [28, 29, 46]. While a recent study in which these distinct striatal pathways were optogenetically manipulated in behaving mice has supported this basic framework [47], other findings suggest a more complicated picture. For example both direct and indirect pathways can be coactivated during movement initiation [49], and Parkinsonism is not reliably associated with increased activity of BG outputs, nor a corresponding decrease in the activity of target thalamocortical networks [5052]. For more extensive discussion of BG pathophysiology, see [53-55].

Another clear prediction of disinhibition and gating is that increased thalamic activity at movement onsets should be produced, or at least permitted, by a corresponding decrease in pallidal firing [56]. However, in contrast to this prediction, studies of pallidal activity in behaving animals have reported that most pallidal neurons also exhibit rate increases at movement onsets [20,24, 57-62], often in the exact same behavioral tasks where thalamic neurons exhibit rate increases. Thus pallidal neurons and the thalamic neurons they inhibit appear to exhibit 'paradoxical co-activation' at movement onset (Figure 2C). One possible explanation for this finding is that pallidal and thalamic neurons exhibiting rate increases could be in different motor 'channels.' In other words, it is possible that pallidal neurons exhibiting a rate increase could cause the firing rate decrease observed in a small fraction of thalamic neurons [52, 55], and the minority of pallidal neurons exhibiting a firing rate decrease could cause the firing rate increase observed in most thalamic neurons. $[33,34,36$, 56]. Another possibility is that pallidal and thalamic activity within a single motor channel really are coactive, but that some mechanism, beyond the simple view of GABA-mediated synaptic inhibition, permits or drives thalamic neurons to spike in the presence of increased pallidal activity at movement onset.

\section{The Rebound Mechanism}

The best way to understand the relation between thalamic activity and its pallidal inputs is to record simultaneously from a thalamic neuron and its presynaptic pallidal input. This was first accomplished in anesthetized songbirds, where simultaneous pallido-thalamic recordings are possible because each thalamic neuron is innervated by a single pallidal axon terminal large enough to be recorded extracellularly [65]. Thus, both pallidal axon and thalamic somatic spikes can be observed at the end of the same electrode. In anesthetized birds it was observed that auditory playback of birdsong causes an increase in the firing rates of both thalamic neurons and their connected presynaptic pallidal input [65]. Thalamic spiking was preceded by rapid acceleration-deceleration of pallidal firing rate, and occurred even after blockade of glutamatergic synaptic transmission in DLM [58, 59], suggesting that thalamic spiking was driven by intrinsic conductances interacting with the fluctuating pallidal inputs $[57,59,60]$. Indeed, studies in brain slice directly showed that pallidal rate increases caused thalamic hyperpolarization and de-inactivation of thalamic low threshold T-type calcium channels, which in turn drove post-inhibitory rebound spikes during a subsequent pallidal pause [66]. Thalamic activity was driven, counterintuitively, by the inhibitory pallidal inputs themselves. Moreover, Person and Perkel (2005) showed that pallidal IPSPs can drive rebound spiking at rates above $15 \mathrm{~Hz}$ and with pallido-thalamic spike latencies shorter than 15 milliseconds at temperatures considerably below body 
temperature [66]. Our biophysical model of this pallidothalamic interaction with kinetics and conductances scaled up to songbird body temperature $\left(41^{\circ} \mathrm{C}\right)$ demonstrated rebound spiking at nearly $30 \mathrm{~Hz}$ when given a realistic pattern of pallidal input in the absence of any glutamatergic input [69]. Thus 'rebound mode' of thalamic activation could in principle contribute to thalamic activation during pallidal rate increases [65].

\section{Cortical activation of BG-recipient thalamus}

In the rebound model, thalamic premotor signals are primarily patterned by pallidal inputs. In contrast to this prediction, however, thalamic onset signals during primate reaching [70] and during songbird vocal babbling [24] persist after ablation of pallidal inputs, suggesting that non-pallidal inputs may be sufficient to drive the thalamic premotor activity (Figure 2D). Songbirds, like mammals, also have a direct cortical projection to the BG-recipient thalamus [62, 63], and we hypothesized that this projection may drive song-locked thalamic signals. Recordings in the singing bird revealed that antidromically-identified corticothalamic neurons exhibited the exact same pattern of firing-rate increases prior to syllable onsets as thalamic neurons (Figure 2D, blue trace). Furthermore, both cortical and downstream thalamic neurons exhibited a dramatic increase in firing rate during singing compared to periods of non-singing [24]. These and other experiments demonstrated that cortical inputs could drive thalamic premotor signals, and at least in the songbird, explained how thalamic neurons acquire syllable-related modulation. Specifically, during the transition to singing and also immediately prior to syllable onsets, thalamic neurons, their inhibitory pallidal inputs, and their excitatory cortical inputs all increased their firing rates at the same time (Figure 2E).

These findings highlight the puzzling nature of BG output. Why do pallido-thalamic coactivations occur? Do they occur in the same BG-thalamic 'channel?' What effect does balanced pallidal inhibition and cortical excitation have on thalamic spiking? Addressing these questions requires examination of the pallidothalamic interaction on a fast, spike-byspike basis within synaptically-connected pairs of pallidal and thalamic neurons. It also requires studying this interaction across different levels of corticothalamic activation. To achieve this, we recently recorded from pallido-thalamic connected pairs in awake, singing and non-singing birds [24, 69] (Figure 3). During non-singing epochs, thalamic neurons fired at high rates $(\sim 60 \mathrm{~Hz})$, while their pallidal inputs discharged at rates of $\sim 150 \mathrm{~Hz}$. Remarkably, in the few hundred milliseconds prior to the onset of a song bout, these thalamic neurons increased their firing rate to roughly $100 \mathrm{~Hz}$, even while the synapticallyconnected pallidal input ramped up to over $250 \mathrm{~Hz}$ firing rate. In addition to this pallidothalamic coactivation at the transition from singing to non-singing, a similar phenomenon was observed at syllable onsets (Figure 2E). As described earlier, all thalamic (DLM) neurons recorded during subsong 'babbling' exhibited a brisk rate increase prior to syllable onsets (Figure 2B) and nearly all pallidal inputs to DLM exhibited a coincident syllableonset peak (Figure 2C). Notably, no DLM neurons exhibited a decrease in activity at syllable onsets. Clear paradoxical coactivation of pallidal inputs with their thalamic targets was observed even in cases where these signals were recorded simultaneously as a synaptically-connected pair. These findings conclusively demonstrated that coactivation of pallidal and thalamic neurons can indeed occur within the same pallidothalamic motor 'channel'.

\section{High Frequency Entrainment of thalamic neurons by basal ganglia outputs}

How does this co-activation occur? Why don't pallidal inputs more effectively suppress thalamic spiking? Do pallidal inputs really produce an effective inhibition of thalamic neurons, or do they contribute to increased thalamic spiking, perhaps by the post-inhibitory 
rebound mechanism observed in anesthetized birds? Close examination of the connected pallidal and thalamic spike trains recorded in singing birds answered these questions, ruled out the rebound mechanism and revealed several novel aspects of the pallidothalamic interaction. First, pallidal inputs were strongly inhibitory. Each pallidal spike resulted in a powerful pulse of inhibition that completely suppressed thalamic spiking (Figure 3B-C). The duration of this inhibitory pulse was extremely brief ( $\sim 5 \mathrm{~ms})$ and relatively fixed, such that thalamic spikes were reliably time-locked to the preceding pallidal spike (Figure $3 \mathrm{C}$ ).

If a pallidal interspike interval is too short - that is, if the next pallidal spike arrives before the end of the inhibitory pulse - then the thalamic neuron cannot spike in that interval. If the next pallidal spike arrives just after the end of the inhibitory pulse, then the thalamic neuron generates a single spike at a precise time relative to the preceding pallidal spike. This effectively sets a well-defined 'threshold' for the pallidal ISI duration above which the thalamic neuron is permitted to spike (blue arrow, Figure 3C). For pallidal ISIs much longer than the inhibitory pulse, the thalamic neuron can spike multiple times, and we found that this discharge happens at fixed intervals until the next pallidal spike arrives. In other words, thalamic neurons fire at relatively fixed rates within pallidal ISIs. In the singing bird, the duration of the inhibitory pulse and the typical pallidal interspike interval were closely matched, such that thalamic neurons typically generated spikes within a significant fraction $(\sim 20 \%)$ of pallidal ISIs. The high rate of thalamic spiking, together with the precise temporal locking of the thalamic spikes to the pallidal spike train suggested the terminology 'high-frequency entrainment' (HFE) for this mode of pallido-thalamic interaction [69].

Importantly, neither the duration of the pallidal inhibitory pulse, nor the rate of subsequent thalamic discharge within a pallidal ISI, exhibited any dependence on neural activity preceding the most recent pallidal spike. This lack of history dependence is important for several reasons. First, because of the slow voltage-dependent kinetics of T-type calcium channels, it is not compatible with rebound spiking, which depends strongly on the prior modulations in pallidal firing rates. Second, it suggests that each pallidal spike 'resets' the thalamic neuron, erasing the impact of recent neural events. It follows that each pallidal ISI functions as a discrete 'unit' of BG output.

We suggest that the essence of the BG-thalamic interaction in the behaving bird can be described by the relation between the number of thalamic spikes that discharges within a pallidal interspike interval, as a function of duration of that interval. We find that this relation is simply a threshold-linear function of the pallidal ISI duration (Figure 3D) [69]. The $\mathrm{x}$-intercept of the relation is the 'threshold' pallidal ISI duration set by the pulse of pallidal inhibition. The slope of the linear relationship is set by the rate at which thalamic spikes tonically discharge within pallidal ISIs (Figure 3D).

Comparing the timescales of the pallidothalamic interaction across singing and nonsinging illustrates how excitatory inputs to the thalamus (from cortical areas and perhaps elsewhere) modulate the thalamic response within pallidal ISIs. During singing, thalamic neurons recovered from the pulse of pallidal inhibition more rapidly than during non-singing, and thus the 'threshold' pallidal ISI duration at which the thalamic neuron begins to spike was lower (shifting the $x$-intercept to the left, Figure $3 \mathrm{G}$ ). Greater excitatory inputs during singing also increased the rate of thalamic tonic discharge within each pallidal ISI, increasing the slope of the relation (Figure 3G).

Together, these findings suggest that the three modes of BG-thalamic interaction: gating, rebound and entrainment are simply determined by the amount of excitation that the thalamic neuron receives. To test this idea, we constructed a biophysically inspired thalamic neuron and simulated the thalamic response to a pallidal spike train in three conditions of 
excitatory input: zero, intermediate and high levels of glutamate conductance. By changing this one parameter, we were able to capture the essential features of all BG-thalamic models under consideration: rebound, gating, and entrainment (Box 1).

\section{Box 1}

\section{Motor thalamic integration of BG and cortical inputs: a unified view}

A biophysical simulation of a thalamic neuron exhibits rebound, gating, and entrainment modes of BG-thalamic transmission, depending on the excitatory conductance. Different modes may predominate depending on behavioral state, e.g., rebound mode during sleep and entrainment mode during waking behavior. We illustrate the excitatory control of transmission mode by providing the simulation with the same pattern of GABAergic pallidal input (Box 1A, a single powerful presynaptic unit) while varying the excitatory conductance (intended to represent the action of a large number of weak, asynchronously firing glutamatergic inputs). All thalamic spiking in this figure was derived from simulated thalamic neuron; for more details see (Goldberg et al, 2012).

With no excitation, pallidal IPSPs leaves the cell at a hyperpolarized potential that allows $\mathrm{T}$-type $\mathrm{Ca}^{2+}$ channels to recover from inactivation. During a pallidal pause, depolarization is initially driven by HCN currents, but T-type channels take over as the membrane potential enters their activation range, until the threshold for $\mathrm{Na}^{+}$spikes is reached ('rebound mode,' Box 1B). Rebound spikes occur during long pallidal ISIs and are facilitated by a preceding increase in pallidal firing rate (Box 1B) that increases hyperpolarization and decreases $\mathrm{T}$ inactivation. This feature makes rebound responses depend on the recent history of pallidal input as well as the current level of pallidal inhibition. Because rebound firing depends on hyperpolarization, longer pallidal ISIs will not generate more thalamic spikes beyond the initial burst.

At intermediate levels of glutamatergic drive, thalamic activity remains strongly suppressed by pallidal inhibition but can be driven by excitatory inputs during pallidal pauses ('disinhibition/gating mode,' Box 1B). In contrast to rebound mode, longer pallidal pauses permit more thalamic spikes, and the number of thalamic spikes that discharge in a pause is determined by the strength of glutamatergic input (Box 1B).

The third mode of BG-thalamic transmission, high frequency entrainment (Box 1D), occurs at high levels of glutamatergic inputs when pallidal 'pauses' are no longer necessary for thalamic spiking, such that both pallidal and thalamic neurons fire together at high rates (as in Figure 3). Here, the thalamic membrane potential trajectory is dominated by synaptic currents and is largely stereotyped following pallidal IPSPs. Consequently, we named this mode high frequency entrainment because thalamic spikes become entrained to preceding pallidal spikes with submillisecond precision, and this precision of results from the strength and uniformity of the single powerful IPSP interacting with a very large glutamate conductance. The effect of the glutamatergic conductance (not the pallidal input) is to set the latency of the pallidal inhibition (yellow bar, Figure 3B) and the number of thalamic spikes that occur in each pallidal ISI (i.e. the threshold and slope of the curve in Figure 3G, respectively).

In summary, rebound mode spiking is driven by intrinsic thalamic conductances and occurs at very low levels of thalamic excitation. Disinhibition mode spiking is driven by glutamatergic inputs, but still requires 'release' from pallidal inhibition and occurs during pauses. As excitation increases, pauses are no longer necessary and thalamic spikes become entrained, rather than completely suppressed, by pallidal inputs. Note that there is no discrete boundary between gating and entrainment modes; they represent ends of a continuum of thalamic activation that gives rise to qualitatively different firing patterns. 
Given the extent to which thalamic excitability fluctuates across behavioral states, we hypothesize that all of these modes may be important for organismal function.

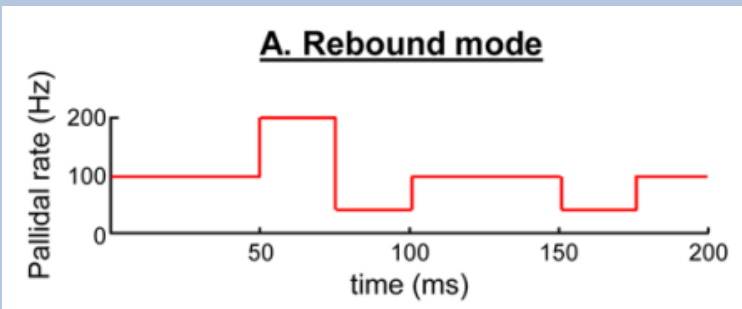

$\mathrm{g}_{\text {glut: }}: 0 \mathrm{nS}$

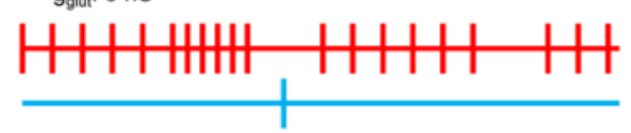

\section{B. Disinhibition/gating mode}

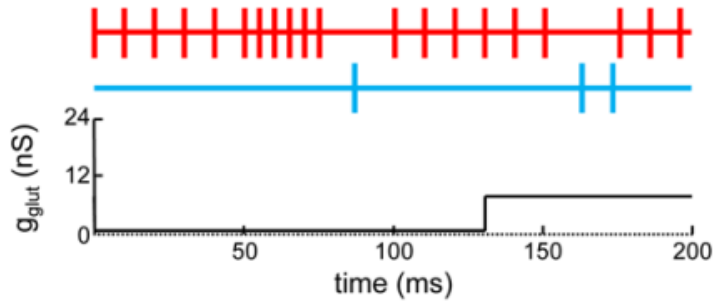

\section{High frequency entrainment mode}
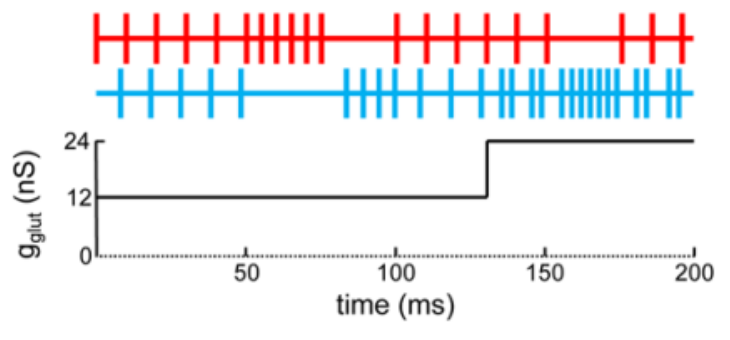

In summary, the BG-recipient thalamus is a critical node within the BG-thalamocortical loop important for motor control and learning. Thalamic neurons frequently exhibit movement onset-related signals, and it has been suggested that pallidal inputs play a primary role in shaping thalamic firing. Paradoxically, pallidal inputs tend to exhibit the same modulations as their thalamic targets, suggesting that they may actually oppose movement-locked thalamic activation, rather than facilitate it (Figure 2). This coactivation does not fit well with current conceptions of the function of BG outputs on motor thalamus, particularly those based on action selection via disinhibition and gating. An important way forward might be to better understand the corticothalamic inputs, which may drive movement related signals and mediate transitions between rebound [65-68], gating [26, 34, 37] and entrainment [24, 69] modes. While the mechanisms underlying thalamic activation have been clarified, the specific information carried by signals converging in the BG-recipient thalamus remains as mysterious as ever. Below we review five key questions that must be resolved to provide a fuller understanding of the organization and functions of this circuit. 


\section{Five open questions of BG -thalamic function}

First, by what mechanisms do pallidal and corticothalamic neurons exhibit similar movement-locked firing patterns? One possibility is that the cortical regions that directly activate the thalamus also indirectly inhibit thalamus by activating BG outputs. For example, in the song system, HVC and LMAN are two cortical premotor nuclei that also exhibit syllable-locked firing, including activations at syllable onsets [73]. These nuclei send an excitatory projection directly to BG outputs and also to the region in the motor cortical nucleus where corticothalamic neurons reside [74, 75] (Figure 1, blue traces; Figure 2E). While the functional anatomy in mammals is more complicated [76], cortical neurons projecting into the hyperdirect pathway can activate BG output neurons via the subthalamic nucleus (STN) [32]. Less well known is that these cortical inputs to the hyperdirect pathway can also project directly to the BG-recipient thalamus [77, 78] and that cortical stimulation simultaneously activates both the thalamic neurons and, through the hyperdirect pathway, their pallidal inputs [79]. Thus, temporally-matched modulation of pallidal and corticothalamic neurons could simply be a natural consequence of the circuit connectivity. Testing these ideas will require combining novel multi-site recording approaches [80-82], with antidromic or optogenetic identification of thalamus-projecting neurons in the BG and cortex [83]. These studies may elucidate how signals propagate through multiple pathways through the $\mathrm{BG}$ to the thalamus during behavior.

Second, what are the functions of spike timing in normal BG function? We have suggested that excitation and inhibition are temporally matched in the thalamus, which may ensure control of spike timing $[84,85]$, In singing birds, thalamic spikes are time-locked to pallidal spikes with submillisecond precision [24], and in mammals BG neurons exhibit temporally precise spikes in relation to brief population bursts that occur at specific times in behavioral tasks [86, 87]. In addition, BG disease is associated with abnormal oscillations and network synchrony [54, 55, 88, 89], but the link between abnormal timing and motor dysfunction remains unclear. Future studies are required to test by what mechanism spike timing could be important. One possibility is that thalamo-recipient cortical neurons are sensitive to synchrony in their thalamic inputs [90,91], in which case the BG could control which ensembles of thalamic neurons are synchronous at any given time, which could in turn determine which cortical neurons are activated from one brief moment to the next. Of course, it is also possible that cortical neurons are simply dependent on the firing rates of their thalamic inputs. To test these and other hypotheses, it will be necessary to record from and manipulate populations of cortical neurons and their thalamic inputs to assess what types of firing patterns propagate to target structures.

Third, what functions are served by the convergence of multiple pallidal inputs to single thalamic neurons? In mammals each neuron in the BG-recipient thalamus is innervated by several pallidal inputs $[105,106]$, whereas in songbirds each DLM neuron is innervated by a single pallidal 'calyceal' axon terminal [107]. This may explain why thalamic neurons discharge at lower rates in mammals than in songbirds-because thalamic firing might be more strongly suppressed by the joint action of convergent pallidal inputs. Convergence of multiple pallidal inputs also suggests that thalamic firing in mammals may not be as dependent on the duration of individual pallidal ISIs. Instead, thalamic spiking may be sensitive to the time elapsed since a spike in any of its pallidal inputs. If this is the case, then synchronization of pallidal activity will have a substantial impact on thalamic spiking and even on the thalamic firing mode, independent of pallidal firing rate [101]. This may explain why pathological synchronization of BG outputs, such as in Parkinsonism, is associated with abnormal thalamic firing and behavior [98]. Further exploration of how DBS influences pallidal synchronization and thalamic firing is necessary. 
Fourth, how do the different BG-thalamic firing modes (Box 1) relate to disease pathogenesis? For example, BG-recipient thalamus is the target of several neuromodulatory systems, including dopamine, and these inputs are lost in Parkinson's [92-94]. Because neuromodulators powerfully regulate thalamic excitability [95], they are poised to influence thalamic membrane potential and thus the mode of BG-thalamic transmission. For example, thalamic rebound bursting is associated with low cholinergic and catecholaminergic tones which occur during low arousal states such as drowsiness or sleep [95, 96], and rebound-like bursts are observed in the BG-recipient thalamus of drowsy animals [97], but less-so during behavioral tasks [18-20]. This suggests an underappreciated importance of neuromodulators acting in the motor thalamus to control motor function. Interestingly, abnormal, synchronized rebound burst events are also observed in pairs of thalamic neurons recorded in Parkinsonian animals $[98,99]$, and theoretical studies suggest that abnormal rebound mode spiking may result in akinesia, tremor, and reduce the fidelity of corticothalamocortical transmission [44, 100-104]. If neuromodulators can indeed switch the thalamus between rebound, gating and entrainment modes, then they could constrain how the BG influences behavior, and could contribute to disease states. Given their potential importance, remarkably little is known about the functions of neuromodulation in BGrecipient thalamus. Simple experiments in brain slice could identify the neuromodulators that regulate thalamic neurons, and these experiments could be followed up with manipulation of neuromodulator levels in the thalamus during behavior.

Finally, what are the specific functions of the cortical projections to the BG-recipient thalamus? This projection remains mysterious even though functions of circuits upstream of BG outputs are relatively well understood (see [108-110]). In a variety of model systems, including songbirds and humans, BG lesions block the behavioral improvements that normally come with practice $[108,111,112]$, and it is widely believed that the BG implement reinforcement learning to mediate the acquisition of motor skills $[8,113,114]$. More specifically, there is evidence that the BG provides the thalamus with a premotor signal that biases motor output towards improved performance $[8,62,110,115-118]$. The end result of learning is, in essence, taking the right action in the right context (or at the right time in a motor sequence) [119], and most models of BG-dependent learning have focused on the role of dopamine-dependent plasticity on striatal medium spiny neurons $[109,120$, 121]. Within this framework, we hypothesized that striatal MSNs integrate contextual (timing), efference copy and reward signals to learn to bias the production of the appropriate vocal gesture at the appropriate time in the song, and the same model was extended to explain stimulus-response learning in mammals [122]. Yet strikingly, to our knowledge, no models of BG-dependent learning incorporate a specific function for the corticothalamic projection. One possibility is that it provides an additional contextual signal about ongoing motor output. For example, premotor bias in birdsong can be generated at precise times in the song sequence [123], and the corticothalamic projection contains song timing information [24] that could exert temporal control over motor output [124]. The corticothalamic projection might also contain an efference copy signal important for learning [124]. Yet another possibility is that the corticothalamic projection controls the gain at which thalamus-dependent behaviors are expressed. For example, in the songbird, the thalamic nucleus DLM is required for the active generation of vocal variability [23]. Thus, if the cortical inputs to DLM are efference copy of descending motor signals, then they could ensure that the amount of variability generated by DLM (and/or its cortical target nucleus LMAN) is commensurate with the demands of the ongoing behavior. For example, the amount of variability injected into a tennis forehand must be greater than a ping pong forehand. Clearly, future studies in both songbirds and mammals will help to test these and other hypotheses. An important first step will be to directly manipulate the activity of corticothalamic projections during behavior to examine its influence over motor variability and learning. 


\section{Acknowledgments}

Funding to MSF was provided by the NIH (grant \# R01DC009183). Funding to JHG was provided by the NIH (grant \# K99NS067062), and the Charles King Trust and Damon Runyon Research Foundation post-doctoral fellowships, and to MAF by the NIH (grant \# NS047985).

\section{References}

1. Kultas-Ilinsky K, et al. Nigrothalamic pathway in the cat demonstrated by autoradiography and electron microscopy. Exp Brain Res. 1978; 33:481-492. [PubMed: 83240]

2. Swanson LW. Cerebral hemisphere regulation of motivated behavior. Brain Res. 2000; 886:113164. [PubMed: 11119693]

3. Parent M, Parent A. The pallidofugal motor fiber system in primates. Parkinsonism Relat Disord. 2004; 10:203-211. [PubMed: 15120094]

4. Schell GR, Strick PL. The origin of thalamic inputs to the arcuate premotor and supplementary motor areas. J Neurosci. 1984; 4:539-560. [PubMed: 6199485]

5. Nambu A, et al. Projection on the motor cortex of thalamic neurons with pallidal input in the monkey. Exp Brain Res. 1988; 71:658-662. [PubMed: 3416976]

6. Redgrave P, et al. The basal ganglia: a vertebrate solution to the selection problem? Neuroscience. 1999; 89:1009-1023. [PubMed: 10362291]

7. Grillner S, et al. Mechanisms for selection of basic motor programs--roles for the striatum and pallidum. Trends Neurosci. 2005; 28:364-370. [PubMed: 15935487]

8. Hikosaka O, et al. Basal ganglia orient eyes to reward. J Neurophysiol. 2006; 95:567-584. [PubMed: 16424448]

9. Marsden CD, Obeso JA. The functions of the basal ganglia and the paradox of stereotaxic surgery in Parkinson's disease. Brain. 1994; 117 (Pt 4):877-897. [PubMed: 7922472]

10. Nambu A. Seven problems on the basal ganglia. Curr Opin Neurobiol. 2008; 18:595-604. [PubMed: 19081243]

11. Reiner, A. The Basal Ganglia IX. Springer; New York: 2009. You cannot have a vertebrate brain without a basal ganglia; p. 3-24.

12. Stephenson-Jones M, et al. Evolution of the basal ganglia; Dual output pathways conserved throughout vertebrate phylogeny. J Comp Neurol. 2012

13. Nadeau SE, Crosson B. Subcortical aphasia. Brain Lang. 1997; 58:355-402. discussion 418-323. [PubMed: 9222518]

14. Carrera E, Bogousslavsky J. The thalamus and behavior: effects of anatomically distinct strokes. Neurology. 2006; 66:1817-1823. [PubMed: 16801643]

15. Canavan AG, et al. Motor learning in monkeys (Macaca fascicularis) with lesions in motor thalamus. Exp Brain Res. 1989; 77:113-126. [PubMed: 2792254]

16. Lorincz E, Fabre-Thorpe M. Effect of pairing red nucleus and motor thalamic lesions on reaching toward moving targets in cats. Behav Neurosci. 1997; 111:892-907. [PubMed: 9383512]

17. van Donkelaar $\mathrm{P}$, et al. Temporary inactivation in the primate motor thalamus during visually triggered and internally generated limb movements. J Neurophysiol. 2000; 83:2780-2790. [PubMed: 10805676]

18. Strick PL. Activity of ventrolateral thalamic neurons during arm movement. J Neurophysiol. 1976; 39:1032-1044. [PubMed: 824408]

19. Anderson ME, Turner RS. Activity of neurons in cerebellar-receiving and pallidal-receiving areas of the thalamus of the behaving monkey. J Neurophysiol. 1991; 66:879-893. [PubMed: 1753292]

20. Kunimatsu J, Tanaka M. Roles of the primate motor thalamus in the generation of antisaccades. J Neurosci. 2010; 30:5108-5117. [PubMed: 20371831]

21. Schlag-Rey M, Schlag J. Visuomotor functions of central thalamus in monkey. I. Unit activity related to spontaneous eye movements. J Neurophysiol. 1984; 51:1149-1174. [PubMed: 6737026]

22. Neafsey EJ, et al. Preparation for movement in the cat. II. Unit activity in the basal ganglia and thalamus. Electroencephalogr Clin Neurophysiol. 1978; 44:714-723. [PubMed: 78800] 
23. Goldberg JH, Fee MS. Vocal babbling in songbirds requires the basal ganglia-recipient motor thalamus but not the basal ganglia. Journal of Neurophysiology. 2011; 105:2729-2739. [PubMed: 21430276]

24. Goldberg JH, Fee MS. A cortical motor nucleus drives the basal ganglia-recipient thalamus in singing birds. Nature Neuroscience. 2012; 15:620-627.

25. MacLeod NK, et al. Evidence for a GABAergic nigrothalamic pathway in the rat. II. Electrophysiological studies. Exp Brain Res. 1980; 40:55-61. [PubMed: 6252030]

26. Tanibuchi I, et al. Substantia nigra output to prefrontal cortex via thalamus in monkeys. I. Electrophysiological identification of thalamic relay neurons. J Neurophysiol. 2009; 102:29332945. [PubMed: 19692504]

27. Anderson ME, et al. Effects of high-frequency stimulation in the internal globus pallidus on the activity of thalamic neurons in the awake monkey. J Neurophysiol. 2003; 89:1150-1160. [PubMed: 12574488]

28. Alexander GE, Crutcher MD. Functional architecture of basal ganglia circuits: neural substrates of parallel processing. Trends Neurosci. 1990; 13:266-271. [PubMed: 1695401]

29. Albin RL, et al. The functional anatomy of basal ganglia disorders. Trends Neurosci. 1989; 12:366-375. [PubMed: 2479133]

30. DeLong MR. Primate models of movement disorders of basal ganglia origin. Trends Neurosci. 1990; 13:281-285. [PubMed: 1695404]

31. Nambu A. A new dynamic model of the cortico-basal ganglia loop. Prog Brain Res. 2004; 143:461-466. [PubMed: 14653188]

32. Frank MJ. Hold your horses: a dynamic computational role for the subthalamic nucleus in decision making. Neural Netw. 2006; 19:1120-1136. [PubMed: 16945502]

33. Deniau JM, Chevalier G. Disinhibition as a basic process in the expression of striatal functions. II. The striato-nigral influence on thalamocortical cells of the ventromedial thalamic nucleus. Brain Res. 1985; 334:227-233. [PubMed: 3995318]

34. Mink JW. The basal ganglia: focused selection and inhibition of competing motor programs. Prog Neurobiol. 1996; 50:381-425. [PubMed: 9004351]

35. Gurney K, et al. A computational model of action selection in the basal ganglia. I. A new functional anatomy. Biol Cybern. 2001; 84:401-410. [PubMed: 11417052]

36. Hikosaka O. GABAergic output of the basal ganglia. Prog Brain Res. 2007; 160:209-226. [PubMed: 17499116]

37. Rouiller EM, et al. Cerebellothalamocortical and pallidothalamocortical projections to the primary and supplementary motor cortical areas: a multiple tracing study in macaque monkeys. J Comp Neurol. 1994; 345:185-213. [PubMed: 7523459]

38. Tracey DJ, et al. Thalamic relay to motor cortex: afferent pathways from brain stem, cerebellum, and spinal cord in monkeys. J Neurophysiol. 1980; 44:532-554. [PubMed: 7441314]

39. Ilinsky IA, Kultas-Ilinsky K. An autoradiographic study of topographical relationships between pallidal and cerebellar projections to the cat thalamus. Exp Brain Res. 1984; 54:95-106. [PubMed: 6698151]

40. Buford JA, et al. Contrasting locations of pallidal-receiving neurons and microexcitable zones in primate thalamus. J Neurophysiol. 1996; 75:1105-1116. [PubMed: 8867121]

41. McFarland NR, Haber SN. Thalamic relay nuclei of the basal ganglia form both reciprocal and nonreciprocal cortical connections, linking multiple frontal cortical areas. J Neurosci. 2002; 22:8117-8132. [PubMed: 12223566]

42. Haber S, McFarland NR. The place of the thalamus in frontal cortical-basal ganglia circuits. Neuroscientist. 2001; 7:315-324. [PubMed: 11488397]

43. Guo Y, et al. Thalamocortical relay fidelity varies across subthalamic nucleus deep brain stimulation protocols in a data-driven computational model. J Neurophysiol. 2008; 99:1477-1492. [PubMed: 18171706]

44. Chevalier G, Deniau JM. Inhibitory nigral influence on cerebellar evoked responses in the rat ventromedial thalamic nucleus. Exp Brain Res. 1982; 48:369-376. [PubMed: 7151930] 
45. Penney JB Jr, Young AB. Speculations on the functional anatomy of basal ganglia disorders. Annu Rev Neurosci. 1983; 6:73-94. [PubMed: 6838141]

46. Gerfen CR. Molecular effects of dopamine on striatal-projection pathways. Trends Neurosci. 2000; 23:S64-70. [PubMed: 11052222]

47. Kravitz AV, et al. Regulation of parkinsonian motor behaviours by optogenetic control of basal ganglia circuitry. Nature. 2010; 466:622-626. [PubMed: 20613723]

48. Chevalier G, Deniau JM. Disinhibition as a basic process in the expression of striatal functions. Trends Neurosci. 1990; 13:277-280. [PubMed: 1695403]

49. Nambu A, et al. Discharge patterns of pallidal neurons with input from various cortical areas during movement in the monkey. Brain Res. 1990; 519:183-191. [PubMed: 2397404]

50. Georgopoulos AP, et al. Relations between parameters of step-tracking movements and single cell discharge in the globus pallidus and subthalamic nucleus of the behaving monkey. J Neurosci. 1983; 3:1586-1598. [PubMed: 6875658]

51. Anderson ME, Horak FB. Influence of the globus pallidus on arm movements in monkeys. III. Timing of movement-related information. J Neurophysiol. 1985; 54:433-448. [PubMed: 4031996]

52. Anderson ME, Turner RS. A quantitative analysis of pallidal discharge during targeted reaching movement in the monkey. Exp Brain Res. 1991; 86:623-632. [PubMed: 1761096]

53. Yoshida A, Tanaka M. Enhanced modulation of neuronal activity during antisaccades in the primate globus pallidus. Cereb Cortex. 2009; 19:206-217. [PubMed: 18477689]

54. Sheth SA, et al. Basal ganglia neurons dynamically facilitate exploration during associative learning. J Neurosci. 2011; 31:4878-4885. [PubMed: 21451026]

55. Turner RS, Anderson ME. Pallidal discharge related to the kinematics of reaching movements in two dimensions. J Neurophysiol. 1997; 77:1051-1074. [PubMed: 9084582]

56. Berns GS, Sejnowski TJ. A computational model of how the basal ganglia produce sequences. J Cogn Neurosci. 1998; 10:108-121. [PubMed: 9526086]

57. Person AL, Perkel DJ. Pallidal neuron activity increases during sensory relay through thalamus in a songbird circuit essential for learning. J Neurosci. 2007; 27:8687-8698. [PubMed: 17687046]

58. Person AL, Perkel DJ. Unitary IPSPs drive precise thalamic spiking in a circuit required for learning. Neuron. 2005; 46:129-140. [PubMed: 15820699]

59. Leblois A, et al. Millisecond timescale disinhibition mediates fast information transmission through an avian basal ganglia loop. J Neurosci. 2009; 29:15420-15433. [PubMed: 20007467]

60. Kojima S, Doupe AJ. Activity propagation in an avian basal ganglia-thalamocortical circuit essential for vocal learning. J Neurosci. 2009; 29:4782-4793. [PubMed: 19369547]

61. Inase M, et al. Changes in the control of arm position, movement, and thalamic discharge during local inactivation in the globus pallidus of the monkey. J Neurophysiol. 1996; 75:1087-1104. [PubMed: 8867120]

62. Wild JM. Descending projections of the songbird nucleus robustus archistriatalis. J Comp Neurol. 1993; 338:225-241. [PubMed: 8308169]

63. Vates GE, et al. Reafferent thalamo- "cortical" loops in the song system of oscine songbirds. J Comp Neurol. 1997; 380:275-290. [PubMed: 9100137]

64. Goldberg $\mathrm{JH}$, et al. Integration of cortical and pallidal inputs in the basal ganglia-recipient thalamus of singing birds. Journal of Neurophysiology. 2012; 108:1403-1429. [PubMed: 22673333]

65. Aronov D, et al. A specialized forebrain circuit for vocal babbling in the juvenile songbird. Science. 2008; 320:630-634. [PubMed: 18451295]

66. Mooney R, Konishi M. Two distinct inputs to an avian song nucleus activate different glutamate receptor subtypes on individual neurons. Proc Natl Acad Sci U S A. 1991; 88:4075-4079. [PubMed: 11607180]

67. Farries MA, et al. Evidence for "direct" and "indirect" pathways through the song system basal ganglia. J Comp Neurol. 2005; 484:93-104. [PubMed: 15717304]

68. Alexander GE, et al. Parallel organization of functionally segregated circuits linking basal ganglia and cortex. Annu Rev Neurosci. 1986; 9:357-381. [PubMed: 3085570] 
69. Feger J, et al. The projections from the parafascicular thalamic nucleus to the subthalamic nucleus and the striatum arise from separate neuronal populations: a comparison with the corticostriatal and corticosubthalamic efferents in a retrograde fluorescent double-labelling study. Neuroscience. 1994; 60:125-132. [PubMed: 8052406]

70. Kita T, Kita H. The subthalamic nucleus is one of multiple innervation sites for long-range corticofugal axons: a single-axon tracing study in the rat. J Neurosci. 2012; 32:5990-5999. [PubMed: 22539859]

71. Wan XS, et al. Mapping of the motor pathways in rats: c-fos induction by intracortical microstimulation of the motor cortex correlated with efferent connectivity of the site of cortical stimulation. Neuroscience. 1992; 49:749-761. [PubMed: 1279454]

72. Leventhal DK, et al. Basal ganglia beta oscillations accompany cue utilization. Neuron. 2012; 73:523-536. [PubMed: 22325204]

73. Pasupathy A, Miller EK. Different time courses of learning-related activity in the prefrontal cortex and striatum. Nature. 2005; 433:873-876. [PubMed: 15729344]

74. Feingold J, et al. A system for recording neural activity chronically and simultaneously from multiple cortical and subcortical regions in nonhuman primates. J Neurophysiol. 2012; 107:1979_ 1995. [PubMed: 22170970]

75. Bodor AL, et al. Structural correlates of efficient GABAergic transmission in the basal gangliathalamus pathway. J Neurosci. 2008; 28:3090-3102. [PubMed: 18354012]

76. Parent M, et al. Two types of projection neurons in the internal pallidum of primates: single-axon tracing and three-dimensional reconstruction. J Comp Neurol. 2001; 439:162-175. [PubMed: 11596046]

77. Luo M, Perkel DJ. A GABAergic, strongly inhibitory projection to a thalamic nucleus in the zebra finch song system. J Neurosci. 1999; 19:6700-6711. [PubMed: 10414999]

78. Bergman $\mathrm{H}$, et al. Physiological aspects of information processing in the basal ganglia of normal and parkinsonian primates. Trends Neurosci. 1998; 21:32-38. [PubMed: 9464684]

79. Okun M, Lampl I. Instantaneous correlation of excitation and inhibition during ongoing and sensory-evoked activities. Nat Neurosci. 2008; 11:535-537. [PubMed: 18376400]

80. Vogels TP, Abbott LF. Gating multiple signals through detailed balance of excitation and inhibition in spiking networks. Nat Neurosci. 2009; 12:483-491. [PubMed: 19305402]

81. Penney JB Jr, Young AB. GABA as the pallidothalamic neurotransmitter: implications for basal ganglia function. Brain Res. 1981; 207:195-199. [PubMed: 6258730]

82. Howe MW, et al. Habit learning is associated with major shifts in frequencies of oscillatory activity and synchronized spike firing in striatum. Proc Natl Acad Sci U S A. 2011; 108:1680116806. [PubMed: 21949388]

83. Rosin B, et al. Physiology and pathophysiology of the basal ganglia-thalamo-cortical networks. Parkinsonism Relat Disord. 2007; 13(Suppl 3):S437-439. [PubMed: 18267279]

84. Wichmann T, Dostrovsky JO. Pathological basal ganglia activity in movement disorders. Neuroscience. 2011; 198:232-244. [PubMed: 21723919]

85. Brown P, Eusebio A. Paradoxes of functional neurosurgery: clues from basal ganglia recordings. Mov Disord. 2008; 23:12-20. quiz 158. [PubMed: 17999423]

86. Rubin JE, et al. Basal ganglia activity patterns in parkinsonism and computational modeling of their downstream effects. Eur J Neurosci. 2012; 36:2213-2228. [PubMed: 22805066]

87. Konig P, et al. Integrator or coincidence detector? The role of the cortical neuron revisited. Trends Neurosci. 1996; 19:130-137. [PubMed: 8658595]

88. Bruno RM, Sakmann B. Cortex is driven by weak but synchronously active thalamocortical synapses. Science. 2006; 312:1622-1627. [PubMed: 16778049]

89. Freeman A, et al. Nigrostriatal collaterals to thalamus degenerate in parkinsonian animal models. Ann Neurol. 2001; 50:321-329. [PubMed: 11558788]

90. Sanchez-Gonzalez MA, et al. The primate thalamus is a key target for brain dopamine. J Neurosci. 2005; 25:6076-6083. [PubMed: 15987937] 
91. Sadikot AF, Rymar VV. The primate centromedian-parafascicular complex: anatomical organization with a note on neuromodulation. Brain Res Bull. 2009; 78:122-130. [PubMed: 18957319]

92. McCormick DA. Neurotransmitter actions in the thalamus and cerebral cortex and their role in neuromodulation of thalamocortical activity. Prog Neurobiol. 1992; 39:337-388. [PubMed: 1354387]

93. Llinas RR, Steriade M. Bursting of thalamic neurons and states of vigilance. J Neurophysiol. 2006; 95:3297-3308. [PubMed: 16554502]

94. Joffroy AJ, Lamarre Y. Single cell activity in the ventral lateral thalamus of the unanesthetized monkey. Exp Neurol. 1974; 42:1-16. [PubMed: 4207724]

95. Pessiglione $\mathrm{M}$, et al. Thalamic neuronal activity in dopamine-depleted primates: evidence for a loss of functional segregation within basal ganglia circuits. J Neurosci. 2005; 25:1523-1531. [PubMed: 15703406]

96. Llinas RR, et al. Thalamocortical dysrhythmia: A neurological and neuropsychiatric syndrome characterized by magnetoencephalography. Proc Natl Acad Sci U S A. 1999; 96:15222-15227. [PubMed: 10611366]

97. Rubin JE, Terman D. High frequency stimulation of the subthalamic nucleus eliminates pathological thalamic rhythmicity in a computational model. J Comput Neurosci. 2004; 16:211235. [PubMed: 15114047]

98. Lenz FA, et al. Single unit analysis of the human ventral thalamic nuclear group: correlation of thalamic "tremor cells" with the 3-6 Hz component of parkinsonian tremor. J Neurosci. 1988; 8:754-764. [PubMed: 3346719]

99. Pare D, et al. Neuronal basis of the parkinsonian resting tremor: a hypothesis and its implications for treatment. Neuroscience. 1990; 35:217-226. [PubMed: 2199839]

100. Magnin M, et al. Single-unit analysis of the pallidum, thalamus and subthalamic nucleus in parkinsonian patients. Neuroscience. 2000; 96:549-564. [PubMed: 10717435]

101. Atallah HE, et al. Separate neural substrates for skill learning and performance in the ventral and dorsal striatum. Nat Neurosci. 2007; 10:126-131. [PubMed: 17187065]

102. Packard MG, Knowlton BJ. Learning and memory functions of the Basal Ganglia. Annu Rev Neurosci. 2002; 25:563-593. [PubMed: 12052921]

103. Graybiel AM. The basal ganglia: learning new tricks and loving it. Curr Opin Neurobiol. 2005; 15:638-644. [PubMed: 16271465]

104. Suri RE, Schultz W. A neural network model with dopamine-like reinforcement signal that learns a spatial delayed response task. Neuroscience. 1999; 91:871-890. [PubMed: 10391468]

105. Doya K. Reinforcement learning: Computational theory and biological mechanisms. HFSP J. 2007; 1:30-40. [PubMed: 19404458]

106. Lauwereyns J, et al. A neural correlate of response bias in monkey caudate nucleus. Nature. 2002; 418:413-417. [PubMed: 12140557]

107. Joshua M, et al. Encoding of probabilistic rewarding and aversive events by pallidal and nigral neurons. J Neurophysiol. 2009; 101:758-772. [PubMed: 19052110]

108. Joel D, et al. Actor-critic models of the basal ganglia: new anatomical and computational perspectives. Neural Netw. 2002; 15:535-547. [PubMed: 12371510]

109. Andalman AS, Fee MS. A basal ganglia-forebrain circuit in the songbird biases motor output to avoid vocal errors. Proc Natl Acad Sci U S A. 2009; 106:12518-12523. [PubMed: 19597157]

110. Turner RS, Desmurget M. Basal ganglia contributions to motor control: a vigorous tutor. Curr Opin Neurobiol. 2010; 20:704-716. [PubMed: 20850966]

111. Thorndike, EL. Animal Intelligence. Hafner; 1911.

112. Wickens JR, et al. Neural mechanisms of reward-related motor learning. Curr Opin Neurobiol. 2003; 13:685-690. [PubMed: 14662369]

113. Costa RM. Plastic corticostriatal circuits for action learning: what's dopamine got to do with it? Ann N Y Acad Sci. 2007; 1104:172-191. [PubMed: 17435119]

114. Frank MJ. Computational models of motivated action selection in corticostriatal circuits. Curr Opin Neurobiol. 2011; 21:381-386. [PubMed: 21498067]

Trends Neurosci. Author manuscript; available in PMC 2014 December 01. 
115. Fee MS. Oculomotor learning revisited: a model of reinforcement learning in the basal ganglia incorporating an efference copy of motor actions. Front Neural Circuits. 2012; 6:38. [PubMed: 22754501]

116. Charlesworth JD, et al. Learning the microstructure of successful behavior. Nat Neurosci. 2011; 14:373-380. [PubMed: 21278732]

117. Charlesworth JD, et al. Covert skill learning in a cortical-basal ganglia circuit. Nature. 2012; 486:251-255. [PubMed: 22699618]

118. Luo M, Perkel DJ. Intrinsic and synaptic properties of neurons in an avian thalamic nucleus during song learning. J Neurophysiol. 2002; 88:1903-1914. [PubMed: 12364516] 


\section{Highlights}

- We review disinhibition, rebound and entrainment modes of basal ganglia output.

- We propose a unifying framework for three types of basal ganglia-thalamic transmission.

- We propose a resolution to the paradox of pallido-thalamic coactivation.

- Excitatory inputs to the thalamus determine the mode of the thalamic firing.

- Pallidal interspike intervals are discrete units of basal ganglia output. 
$\underline{\text { Mammal }}$

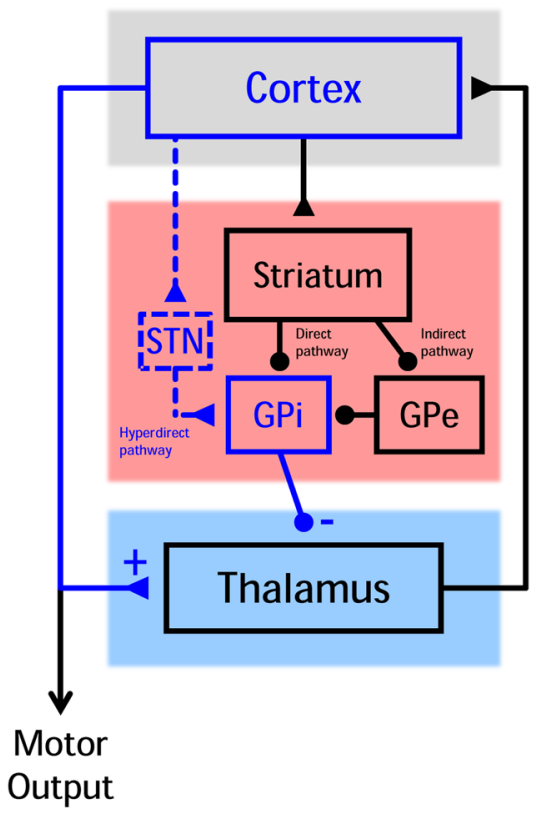

Songbird

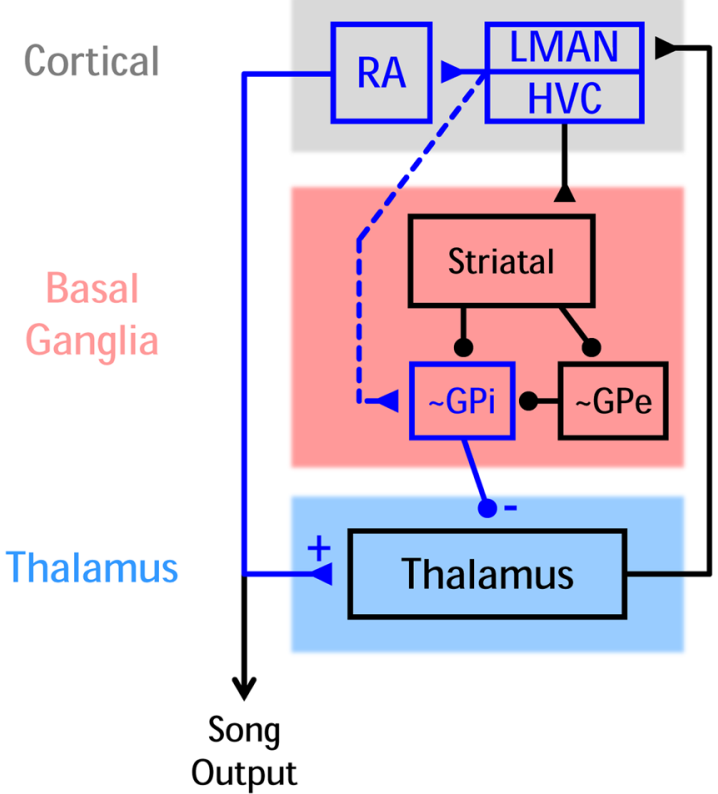

Figure 1. Basal ganglia thalamocortical loops are evolutionarily conserved

In songbirds and mammals, the BG is situated in a thalamocortical loop. Songbirds also have the same major striatal cell classes observed in mammals (not shown) as well as direct and indirect pathways through pallidal neurons analogous to internal and external pallidal segments of primates (GPi and GPe, respectively). Dashed blue lines show hyperdirect pathways connecting cortical areas to BG output (GPi) neurons. This pathway causes inhibition of thalamus. Solid blue lines show pathways connecting cortical areas directly to thalamus. This pathway causes excitation of thalamus. We hypothesize that this subcircuit might ensure that excitation and inhibition of thalamus are temporally matched; these convergent pathways could also drive paradoxical pallido-thalamic co-activations observed across behaviors and model systems. Note that the singing-related portion of the songbird BG lacks a homolog of the mammalian subthalamic nucleus (STN), yet an analogous circuit exists in the form of projections from cortical nuclei LMAN and HVC directly to the $\sim \mathrm{GPi}$ like output neurons. Circles and triangles represent GABAergic and Glutamatergic projections, respectively. 


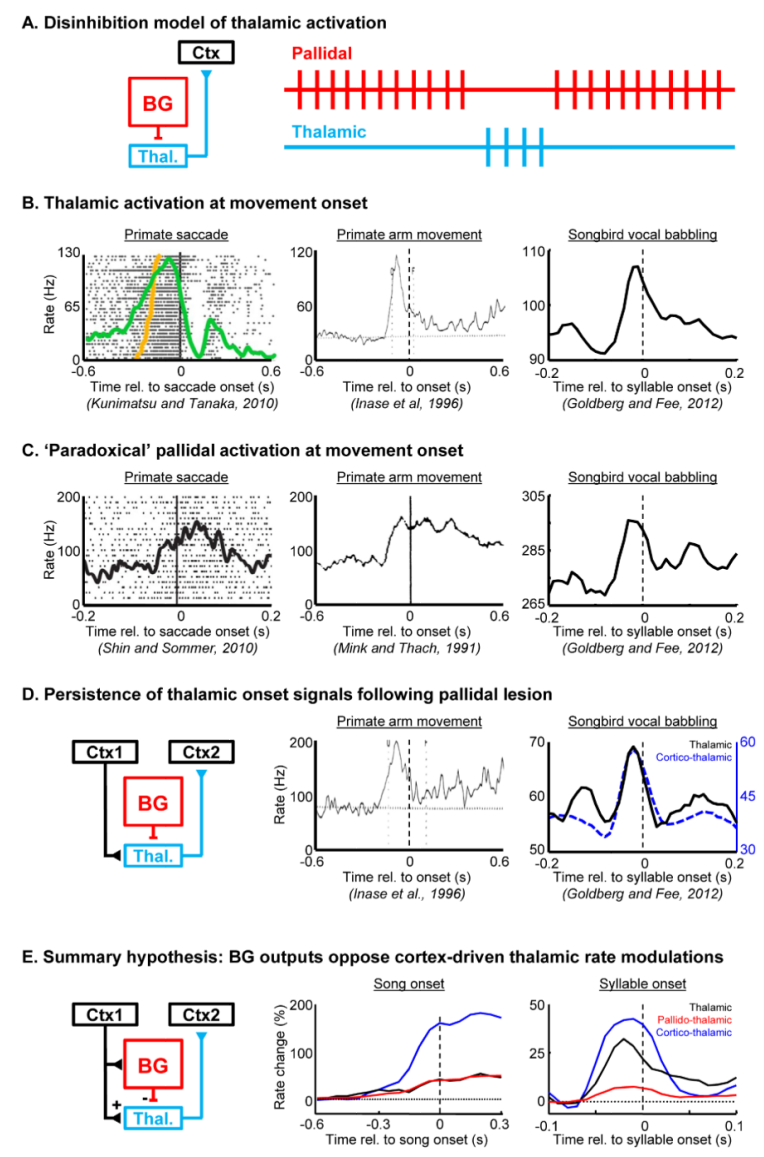

Figure 2. 'Paradoxical' co-activation of thalamic neurons and their inhibitory BG inputs, observed across model systems and behavioral paradigms

(A) Left, the place of the thalamus in a canonical BG-thalamocortical loop. In a predominant view of BG-thalamocortical transmission, thalamic activation occurs during pallidal pauses, through a process of disinhibition. Right, schematic of pallidal and thalamic spike trains (red and blue, respectively), showing thalamic activation during a pause in tonic pallidal firing. (B-C) Movement-onset aligned rate histograms showing thalamic (B) or pallidal (C) activation at movement onset in primates during saccades and arm movements, as well at syllable onset in songbirds during vocal babbling. (D) Left, the BG-recipient thalamus is embedded in a cortico-thalamocortical loop. Movement onset-aligned rate histograms show that thalamic onset signals persist following lesion of pallidal inputs in primates (center) and in songbirds (right). In songbirds, antidromically identified corticothalamic neurons also exhibit rate peaks at syllable onsets. Together, these findings suggest that cortical inputs to the BG-recipient thalamus can drive thalamic pre-motor signals, and further suggest that BG inputs may oppose the cortically driven rate modulation. (E) Left, a schematic of the BGthalamocortical loop highlighting that cortical regions projecting to the $\mathrm{BG}$ can also project to the thalamus $[77,78]$. Rate histograms showing that pallidal (red), thalamic (black) and corticothalamic (blue) neurons exhibit rate increases prior to song onsets (center), as well as syllable onsets (right)(Data from Goldberg and Fee, 2012). Such coactivations might be explained by cortical inputs that project to both the hyperdirect pathway and to the thalamus. Panels in (C-E) are reproduced with permission from (Shin and Sommer, 2010; Mink and Thach, 1991; Goldberg and Fee, 2012; and Inase et al, 1996). 
A
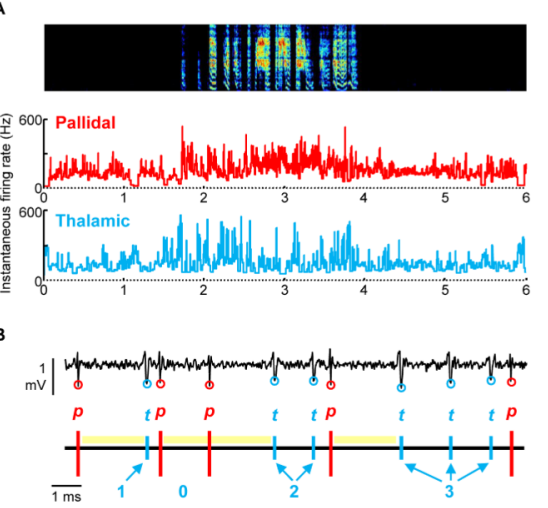

C

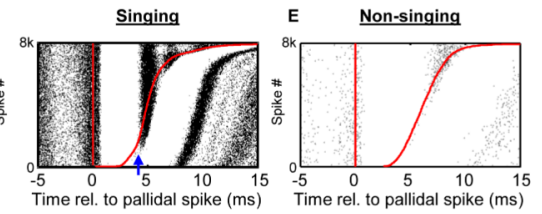

D

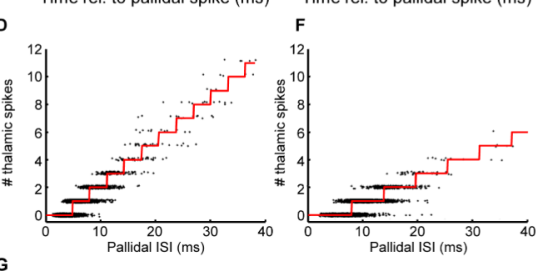

G

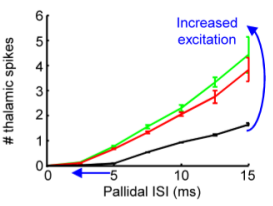

Figure 3. High frequency entrainment of thalamic neurons by pallidal inputs in the singing bird (A) Instantaneous firing rates of a thalamic neuron (blue) and its simultaneously recorded pallidal terminal (red) plotted during a bout of song (spectrogram shown above, dph 45).

Note that both thalamic neuron and its pallidal input fire in concert at more than 100 spikes per second. (B) Example of extracellular waveform containing signals from a thalamic neuron (blue circles) and its presynaptic pallidal axon terminal (red circles). Bottom, schematic of the pallidal and thalamic spike trains. Yellow bars represent periods of thalamic spike suppression following the pallidal spike. The number of thalamic spikes that occurred in each pallidal interspike interval is annotated in blue. (C) Raster plot of thalamic spikes (black ticks) aligned to the timing of pallidal spikes (red line). Red line at right shows the time of the next pallidal spike. The plot is sorted by the duration of the pallidal ISI ( $n=8000$ ISIs during singing). Note that each pallidal spike is followed by a brief period of absolute thalamic spike suppression. (D) The number of thalamic spikes that discharge within a pallidal ISI is plotted as a function the pallidal ISI's duration (data are from singing periods). Red line indicates the number of thalamic spikes predicted to occur based on a simple mathematical model incorporating the pallidal ISI duration and the amount of excitation that the thalamic neuron receives (see Goldberg et al., 2012 for more detail). (EF) Data are plotted as in C-D for non-singing periods, when there is less corticothalamic excitation. (G) The average number of thalamic spikes that discharged within a pallidal ISI is plotted against the duration of that ISI, for three different levels of corticothalamic activation: nonsinging periods (black), and the periods prior to syllable onsets (green) and offsets (red). Excitation of the thalamic neuron has the effect of increasing the slope and 
decreasing the x-intercept of this plot. This figure is adapted from (Goldberg and Fee, 2012; and Goldberg et al, 2012). 